\title{
Wind Power Interval Prediction Based on Improved PSO and BP Neural Network
}

\author{
Jidong Wang ${ }^{\dagger}$, Kaijie Fang*, Wenjie Pang* and Jiawen Sun*
}

\begin{abstract}
As is known to all that the output of wind power generation has a character of randomness and volatility because of the influence of natural environment conditions. At present, the research of wind power prediction mainly focuses on point forecasting, which can hardly describe its uncertainty, leading to the fact that its application in practice is low. In this paper, a wind power range prediction model based on the multiple output property of BP neural network is built, and the optimization criterion considering the information of predicted intervals is proposed. Then, improved Particle Swarm Optimization (PSO) algorithm is used to optimize the model. The simulation results of a practical example show that the proposed wind power range prediction model can effectively forecast the output power interval, and provide power grid dispatcher with decision.
\end{abstract}

Keywords: Wind power prediction, Interval prediction, BP neural network, Improved PSO

\section{Introduction}

Compared with the traditional energy, wind energy has a character of strong randomness, volatility and intermittency. Practical examples show that connecting wind power to power grid would have a unfavorable influence on the security and stable operation of the electrical system [1-2]. Wind power forecasting is one of the most critical technologies to solve wind power undulation. Accurate wind power forecasting can reduce the reserve capacity of power system, lower the operational costs, ease wind power integration impact on power grid and simultaneously provide power grid dispatcher with decision theory [3].

Short-term prediction of wind power is good for the online optimizing operation of electrical power system and can provide power grid dispatcher with basis. The traditional wind power forecasting mainly focuses on time series points, and can just obtain the instantaneous power of a certain moment or the average power in a time slot. Time series [4], principal component analysis (PCA) [5], neural network (NN) [6-9], support vector machine (SVM) [10], wavelet analysis [11] and other methods have been used in short-term wind power prediction. For a single prediction model, large error may appear at some points, literatures [12-14] respectively make combinations of phase space reconstruction, wavelet decomposition, support vector machine and neural network, to build comprehensive prediction models based on a variety of algorithms. These models reduce the occurrence of large error, and improve the prediction accuracy to a certain degree.

$\dagger$ Corresponding Author: Key Laboratory of Smart Grid of Ministry of Education, Tianjin University, China. (jidongwang@tju.edu.cn)

* Key Laboratory of Smart Grid of Ministry of Education, Tianjin University, China. (\{fangkaijie, pangwenjie\}@ tju.edu.cn, jiawensun89@gmail.com)

Received; June 29, 2016 ; Accepted; January 17, 2017
However, the power output of wind power has serious uncertainty due to natural environmental factors impact. Simple point forecast curve of wind power output can hardly describe this uncertainty. Under the circumstance of meeting certain confidence probability, conducting interval forecasting on wind power has an advantage in describing the uncertainty of wind power output power with more practical meaning.

On the aspect of uncertainty analysis, literature [15] defines the risk indices of degree of stability of weather situation measurement for wind power forecast model and describes the uncertainty of online wind power prediction results. Literature [16] presents to describe the uncertainty in wind power forecasting based on the indirect algorithm of probability density function of $\beta$ distribution. Concerning the interval forecasting, the literature [17] employs the empirical distribution model to build the probability-distribution function of the wind power forecast errors. Based on wind power point-forecast values, the literature [18] applies the non-parametric kernel density estimation method to calculate wind power's probability density and then the wind power's prediction intervals are obtained via the fitted probability distribution curve.

The interval prediction methods mentioned above are mostly based on the analysis of forecasting errors distribution features, so the correlative coefficient matrix of forecasting errors, probability density function, joint probability distribution function and the like are required to calculate [19]. Enough data sample and effective nonlinear fitting methods are needed in those methods, and too many calculating steps make those methods complicated.

This paper proposes a wind power range prediction model based on Improved PSO and BP neural network, which utilizes the multiple output property of neural network and directly predicts the top and bottom limitation 
of the power range that accords with a certain probability through historical data. The contribution of this paper is:

(i) The prediction model proposed can avoid complicated nonlinear fitting and obtain predicted results by limited data. On the basis of traditional prediction accuracy, the interval band average bandwidth, average centering error and other parameters are identified to establish a comprehensive objective function.

(ii) PSO algorithm is an effective and widely used method in optimal questions. In this paper, to expand the search range of velocity, we identified a velocity changing coefficient. In this way, the better solution can be obtained by the improved PSO algorithm.

The first part of the paper introduces some recent researches of the prediction wind power. The part 2 explains the principle of BP Neural Network (BPNN) and improved PSO, and modeling of wind power interval prediction is introduced in part 3. Real wind speed data is utilized to test the effectiveness of the forecasting model in part 4 , and the conclusion is shown in part 5.

\section{BPNN and PSO}

\subsection{BPNN}

The BP neural network ${ }^{[20]}$ is a multiple layer feedforward network characterized by forward signal transmission and reverse error spreading. The input signal is successively processed through the input layer and the hidden layer. At the same time, the effect of the neuron on each layer is limited to the neuron on the next layer. Once the output layer doesn't obtain the expected result, the network will automatically adjust the weight and threshold value according to the output error, so as to make the output predicted result gradually approaches the expected value. Its network topology is as shown in Fig. 1.

In the Fig. 1, $\left\{x_{1}, x_{2}, \cdots, x_{n}\right\}$ is the input of the BP neural network, $\left\{y_{1}, y_{2}, \cdots, y_{m}\right\}$ is the predicted output value of the network, $\omega_{i j}$ and $\omega_{j k}$ are weights of the network. For wind power range prediction, let's take the historical data of wind power as the input of the prediction model. And the output results $\left\{y_{1}, y_{2}\right\}$ represent the top and bottom

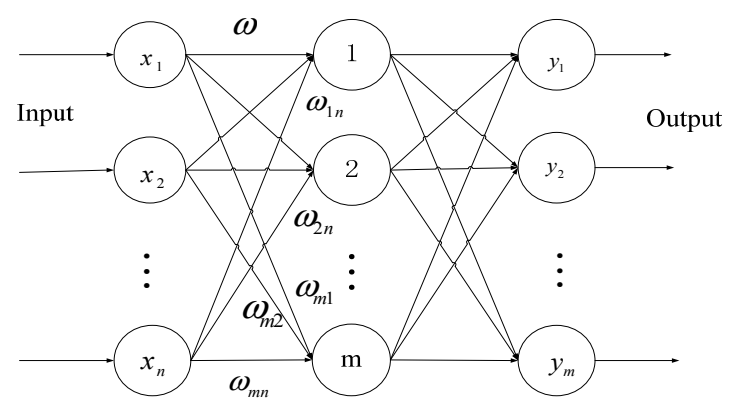

Fig. 1. BP neural network topology limitation of the range output by wind power forecast at the future time nodes. The reliability requirements of the prediction model can be satisfied by adjusting weights and threshold values of the network.

\subsection{PSO}

PSO is a global random searching method based on swarm intelligence, which was proposed through simulating the migratory and swarm behaviors of bird flocks during foraging. In PSO, each particle represents a potential solution to the problem of optimization, the characteristic of which is defined by three indexes, i.e. position, velocity and fitness value. And the fitness value is determined by the fitness function. The particle adjusts its flying dynamically according to its own flying experience as well as the companions', which guides the individual towards optimum within the search-space ${ }^{[21]}$.

Assuming that the search-space dimension of the optimization is $D$, the number of the swarms is $n$, then these particles can be noted as $\omega=\left(\omega_{1}, \omega_{2}, \cdots, \omega_{n}\right)$. The $i^{\text {th }}$ particle represents a vector $\omega_{i}=\left(\omega_{i 1}, \omega_{i 2}, \cdots, \omega_{i D}\right)$ with $D$ dimension. The vector of the particle consists of three parts, i.e. the position of the particle $x_{i}$, the particle's velocity $v_{i}$ and the best individual position known so far $p_{i}$ :

current position: $x_{i}=\left(x_{i 1}, x_{i 2}, \cdots, x_{i D}\right)$;

best position: $p_{i}=\left(p_{i 1}, p_{i 2}, \cdots, p_{i D}\right)$;

particle velocity: $v_{i}=\left(v_{i 1}, v_{i 2}, \cdots, v_{i D}\right)$.

At the same time, noting down the global extremum of the group as $P_{g}=\left(P_{g 1}, P_{g 2}, \cdots, P_{g D}\right)$.

The position and velocity for each particle are updated according to formula (1) and formula (2):

$$
\begin{gathered}
v_{i d}^{k+1}=W \\
\times v_{i d}^{k}+c_{1} \times \operatorname{rand}_{1} \times\left(p_{i d}^{k}-x_{i d}^{k}\right)+c_{2} \\
\times \operatorname{rand}_{2} \times\left(p_{g d}-x_{i d}^{k}\right) \\
x_{i d}^{k+1}=x_{i d}^{k}+v_{i d}^{k+1}
\end{gathered}
$$

Among them, $d=1,2, \cdots, D ; i=1,2, \cdots, n ; 1 \leq k \leq N$; $N$ is the total number of iterations; $c_{1}$ and $c_{2}$ are nonnegative constants, and rand ( ) is a random number among $[0,1]$. $W$ is the inertia weight, which determines the effect of the particle's previous experience on its current velocity.

In the hybrid algorithm, the weight value and threshold value in the BP neural network can be expressed through the particle status in the PSO. Take these two parameters to form a real-valued vector and search for the optimal particle in the given optimization objective function. The shortcomings of PSO include a low accuracy and easily trapping into a local optimum, the reason for which is the update speed of the particle does not have an adaptive time-varying characteristic, and the particle is not able to carry out a detailed and global search.

In order to ensure the diversity of particles in the 
searching space, each individual particle is set with different speeds. The higher speeds meet particles' demand of global optimization, while the lower speeds can confer particles with better local searching ability, so the formula (1) and formula (2) can transform to formula (3):

$$
\begin{cases}v_{i d}^{k+1}=W \times v_{i d}^{k}+c_{1} \times \operatorname{rand}_{1} \times\left(p_{i d}^{k}-x_{i d}^{k}\right)+c_{2} \times \operatorname{rand}_{2} \times\left(p_{g d}-x_{i d}^{k}\right) \\ x_{i d}^{k+1}=x_{i d}^{k}+v_{i d}^{k+1} \\ v_{i d}^{m}=a(m) v_{i d}^{0} \quad m=1,2, \cdots, j \\ x_{i d}^{m}=x_{i d}^{0}+v_{i d}^{m} \quad m=1,2, \cdots, j\end{cases}
$$

Among these, $v_{i d}^{0}$ is called ith particle's standard velocity component in the dimension $d, v_{i d}^{m}$ is called $i^{\text {th }}$ particle's search speed component in the dimension $d$, and $1 \leq j \leq k+1 ; x_{i d}^{0}$ is called $i^{\text {th }}$ particle's reference position component in the dimension $d, x_{i d}^{m}$ is called $i^{\text {th }}$ particle's search position component in the dimension $d ; a(m)$ is called velocity changing coefficient which is used to confirm the relation between two velocities, and the value is confirmed by formula(4):

$$
a(m)=\left\{\begin{array}{cc}
m & v_{i d}^{0}<v_{\min } \\
m / j & v_{i d}^{0} \geq v_{\max } \\
1 \pm m / j & v_{\min }<v_{i d}^{0}<v_{\max }
\end{array}\right.
$$

Inertia weight $W$ affects the searching performance of PSO. The overall searching ability of PSO algorithm increases with $W$ values growth while the local searching ability for optimization is the opposite. Changing the weight $W$ from a fixed value to dynamically decreasing value can optimize the searching progress. The value of $W$ decreases as formula (5):

$$
W(k)=W_{\max }-\frac{W_{\max }-W_{\min }}{N} \times k
$$

where the $W_{\max }$ and $W_{\min }$ are set as the boundary of $W$. $1 \leq k \leq N ; k$ is the number of iterations $N$ is the total number of iterations.

The improvement mentioned above can maintain particle's diversity in searching space. At the same time, the dynamic inertia weight can balance the overall and local searching ability. The algorithm performances are improved in the aspects of convergence speed and searching precision.

\section{Optimization Criterion and Model of Wind Power Interval Prediction}

Power interval prediction is different from traditional point forecasting. Traditional point forecasting is based on the lots of historical data, and has a requirement of real- time feature. The goal of wind power range prediction is to ensure the reliability of predicting outcomes, try to meet the decision demands as much as possible, and improve the practical engineering applicability. Wind power time series is set as $X=\left[x_{1}, x_{2}, \cdots, x_{n}\right] ; U_{i}$ and $L_{i}$ are top and bottom limitations at the moment of $t=i ; N_{t}$ is the sample size employed in calculating interval parameters, whose confidence probability is $\theta=100(1-\alpha) \%$, and $\omega$ is the vector of network weight and threshold parameters.

\subsection{Predicted interval coverage probability (PICP)}

Interval prediction results should be able to meet the demand for reliability and the results can be described through cover probability. As to the sample set whose capacity is $N_{t}$, the probability of actual $x_{i}$ in the forecasting interval $\left[L_{i}, U_{i}\right]$ should be no less than $\theta=100(1-\alpha) \%$. When $x_{i}$ belongs to the interval $\left[L_{i}, U_{i}\right]$, we call the forecasting interval can cover the real value. The PICP is calculated by formula (6):

$$
\begin{gathered}
P I C P=\frac{1}{N_{t}} \sum_{i=1}^{N_{t}} \xi_{i} \\
\xi_{i}= \begin{cases}0 & x_{i} \notin\left[L_{i}, U_{i}\right] \\
1 & x_{i} \in\left[L_{i}, U_{i}\right]\end{cases}
\end{gathered}
$$

\subsection{Predicted interval normalized average width (PINAW)}

Different from point forecast, the interval forecast of wind power does not simply pursue the accuracy of forecasting results. What's more important is to provide decisions for power grid dispatchers with bases, so, forecasting models that meet certain coverage probability demands are acceptable in theory. To this end, considering the PINAW in evaluation criteria, the over-completed risks caused by forecasting interval's bounds being close to its limited value should be lowered. For normalized time series of wind power, PINAW is calculated via formula (8):

$$
P I N A W=\frac{1}{N_{t}} \sum_{i=1}^{N_{t}}\left(U_{i}-L_{i}\right)
$$

\subsection{Predicted interval average centering error (PIACE)}

In order to evaluate the distribution uniformity of actual value in the forecasting interval, the PIACE, which means offset between interval's mid-value and the actual output value, should be considered:

$$
\text { PIACE }=\sqrt{\sum_{i=1}^{N_{t}}\left(x_{i}-\left(U_{i}+L_{i}\right) / 2\right)^{2}}
$$




\subsection{Optimized objective function}

The target of wind power range prediction is to make the coverage probability as high as possible, and at the same time, interval average bandwidth and average centering error should be as small as possible. This is an issue of multi-target optimization which requires integrating every index to establish wind power range prediction parameter consolidated optimized objective function:

$$
\begin{gathered}
F(\omega)=P I N A W \times\left(1+k \times \lambda(P I C P) \times e^{P I A C E}\right) \\
\lambda(P I C P)= \begin{cases}0 & P I C P \geq \theta \\
1 & P I C P<\theta\end{cases}
\end{gathered}
$$

where, the non-negative constant $k$ is called penalty parameter. $k$ is set to 0.5 in this paper according to experience of calculation.

After building consolidated optimal function, we can transfer the wind power range prediction issue into the following optimization issue with restraints:

$$
\text { Min: } F(\omega) \quad \text { s.t. } \quad \theta \leq P I C P(\omega) \leq 100 \%
$$

The steps of building the wind power range prediction model are as follows:

1) Selecting wind power's historical data, and dividing it into training dataset and testing dataset. Then normalizing the original wind power data.

2) Initializing the forecasting model's parameters, including weight, threshold value and the initialized parameter vector $\omega$ of the BP neural network; group

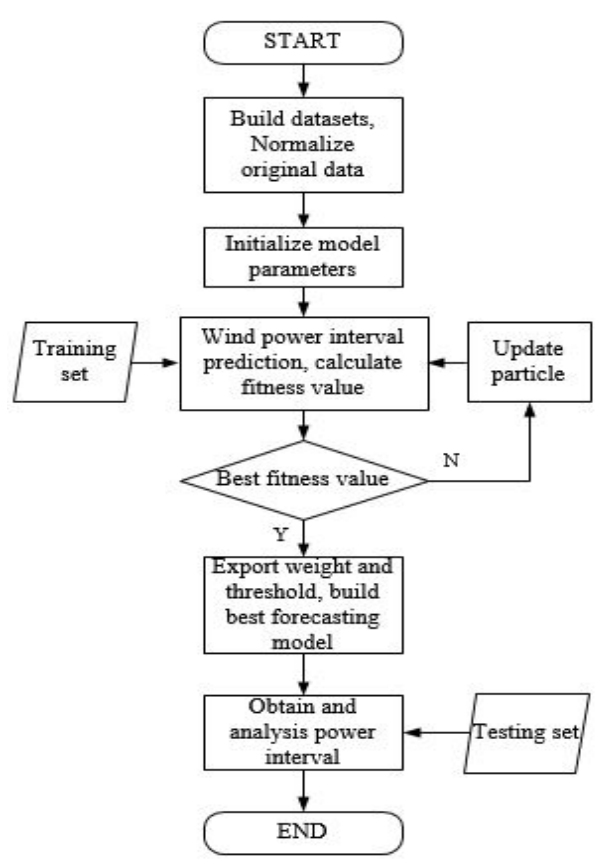

Fig. 2. The process of wind power interval prediction model size, iterations and inertia weight variation interval, and initialized particle position and velocity in particle swarm algorithm.

3) Based on neural network parameter vector $\omega$, conducting neural network forward prediction. Setting the wind power data in the past moment $[t-m, t-m+1, \cdots, t]$ as input; obtaining $t+1$ moment predicted wind power interval bounds, then the value of $F(\omega)$ is calculated via formula (10).

4) Searching and gaining the best particle by improved PSO, and the particle is the best parameter vector of BP neural network.

5) Using the forecasting model with the best network parameters to conduct interval prediction of wind power on the testing dataset, and then carry out error analysis on the results.

The process of wind power interval prediction model is shown in Fig. 2.

\section{Example Analysis}

In this paper, we take measured data from Key Laboratory of Smart Grid of Ministry of Education (Tianjin University) as an example to verify the effectiveness of the algorithm mentioned above in wind power range prediction. The original input shown in Fig. 3 is surrounding wind speed of fan wheel in January 2013. It was measured by wind speed sensors installed closed to fan wheels, and each point represents the average wind speed per hour, which is calculated by four values collected by every 15 minutes.

Regarding the AOC15/50 fan produced by Atlantic Orient as the physical model of computation of wind power; its wind speed-power curve is shown in Fig. 4, the mathematical expression is shown in formula (13), and the original data of wind power obtained after computation is shown in Fig. 5.

$$
P=\left\{\begin{array}{cl}
0 & v<v_{c i}, v>v_{c o} \\
0.0444 v^{2}-1.6114 v^{2}+24.272 v-87.417 & v_{c i} \leq v \leq v_{c r} \\
0.025 v^{2}-1.525 v+82.5 & v_{c r}<v \leq v_{c o}
\end{array}\right.
$$

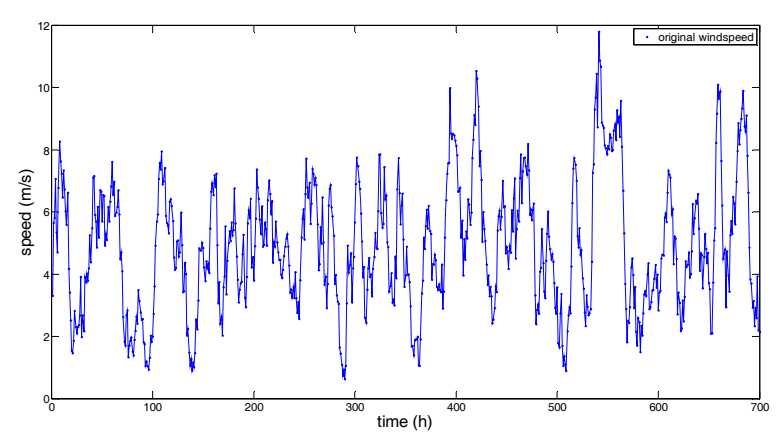

Fig. 3. Original wind speed 


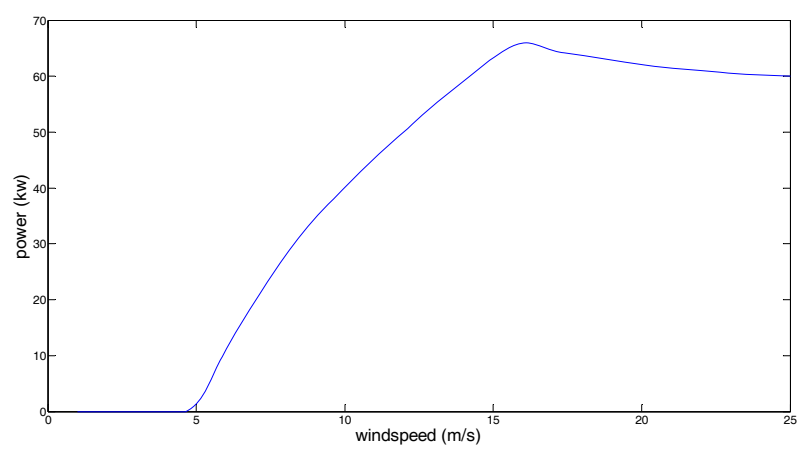

Fig. 4. Wind speed-power curve

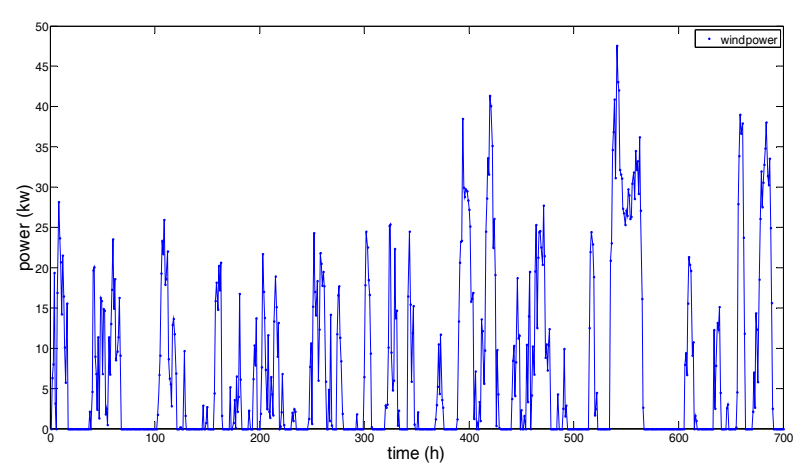

Fig. 5. Wind power data

In the Eq. (13), $P$ refers to the output power; $v$ refers to surrounding air speed around the hub; $v_{c i}$ refers to the startup wind speed; $v_{c r}$ and $v_{c o}$ refer to the rated work wind speed and the cut-off wind speed of the fan respectively.

Wind power data is divided into 10 parts. 6 of them are selected randomly to make up the training set, the other 4 parts is chosen as the testing set. And in this paper, we randomly choose one part of the testing set to show the simulation results. The group size of PSO is set to 20, and the number of iterations is set to 100. Particle's position and velocity are initialized randomly; and inertia weight linearly decreases from 0.9 to 0.4 in terms of iteration algebra.

According to the characteristic of wind speed, a three layer BPNN is designed to predict wind power. The input is historical wind power data, and last five real wind power value is selected in this paper. So the number of neurons for input layer is 5. The output is boundary of predicted wind power, so the neuron number of output layer is 2 . The number of hidden layer is set to 6 by the trial and error.

Select different confidence probability separately $(80 \%$ and $90 \%$ are selected to make a comparison and apply tested data to predict wind power short-term interval; carry out wind power range prediction employing improved PSO-BPNN which is proposed in this article and single neural network respectively. The forecasting results are shown in Fig. 6 and Fig. 7 and forecasting interval's parameters refer to Table 1 .

As can be seen from the Fig. 6 to Fig. 9, the neural
Table 1. Results of short-term wind power interval prediction

\begin{tabular}{c|c|c|c|c|c|c}
\hline \multirow{2}{*}{$\begin{array}{c}\text { Confidence } \\
\text { probability }\end{array}$} & \multicolumn{2}{|c|}{ BPNN } & \multicolumn{2}{c|}{ PSO-BPNN } & \multicolumn{2}{c}{$\begin{array}{c}\text { Improved } \\
\text { PSO-BPNN }\end{array}$} \\
\cline { 2 - 7 } & PIANW & PIACE & PIANW & PIACE & PIANW & PIACE \\
\hline $80 \%$ & 0.1547 & 0.0572 & 0.1135 & 0.0426 & 0.0983 & 0.0379 \\
\hline $90 \%$ & 0.3203 & 0.0916 & 0.2473 & 0.0781 & 0.1943 & 0.0664 \\
\hline
\end{tabular}

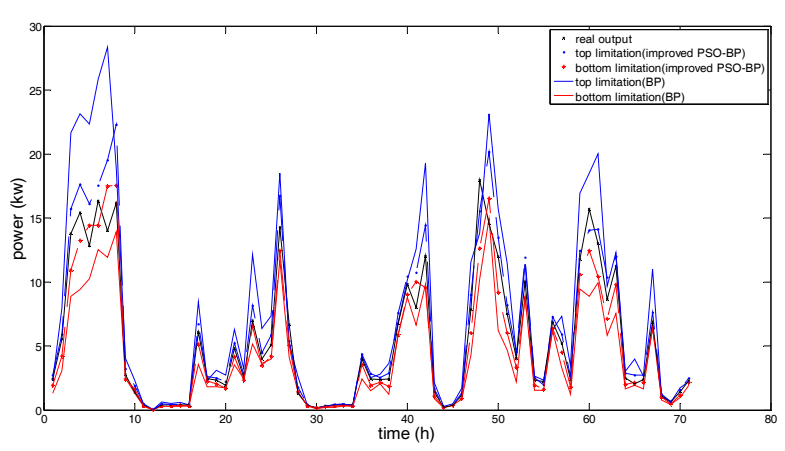

Fig. 6. Results of short-term wind power interval prediction compared with BPNN model $(80 \%$ confidence probability)

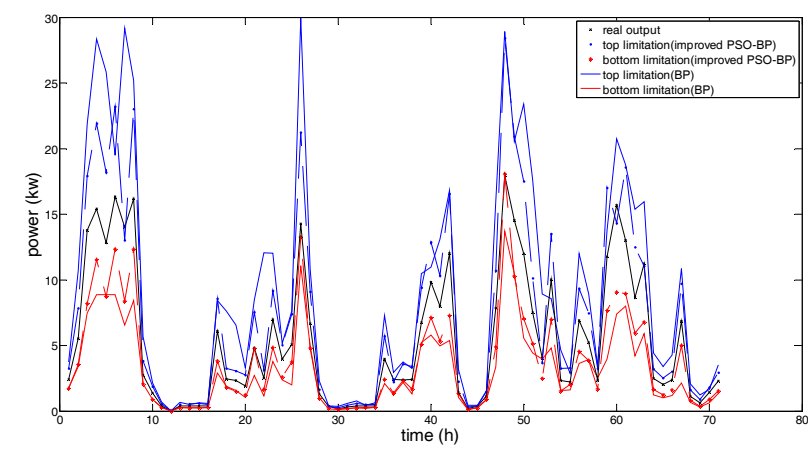

Fig. 7. Results of short-term wind power interval prediction compared with BPNN model $(90 \%$ confidence probability)

network interval prediction model, which is based on improved particle swarm algorithm, can achieve wind power short-term power interval forecasting. By optimizing the PSO search, the PINAW and PIACE of the forecasting results are apparently improved. In terms of the forecasting results, the forecasting accuracy of the improved PSO-BP model is relatively high. When the fan's practical output power is low and the changes are relatively steady, the forecasting interval top and bottom limitations are extremely close to the practical power value and the interval bandwidth is fairly narrow. In the time span existing random fluctuation or abrupt changes, top and bottom limitations can track the trend of electrical power generation variation of the distributed power generation system, but the interval bandwidth is a little wider and prediction accuracy is lower. 


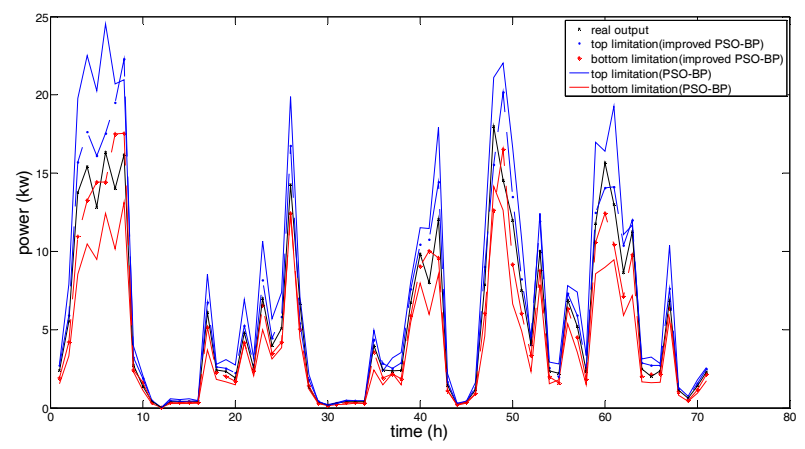

Fig. 8. Results of short-term wind power interval prediction compared with PSO-BP model $(80 \%$ confidence probability)

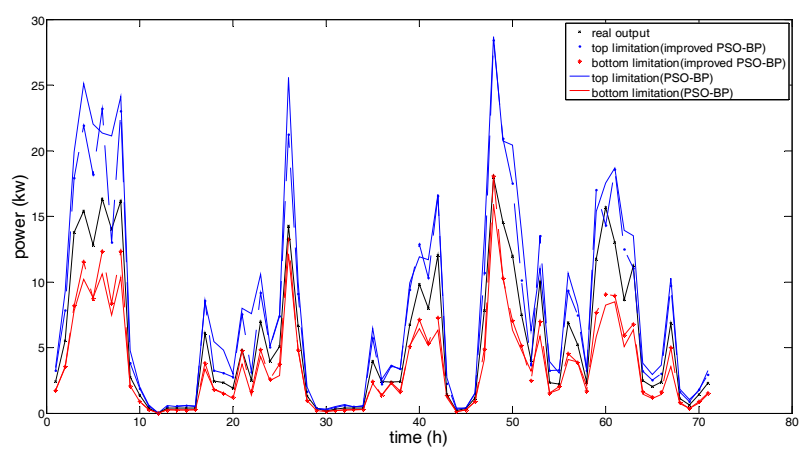

Fig. 9. Results of short-term wind power interval prediction compared with PSO-BP model $(90 \%$ confidence probability)

As is shown in Table 1, when the confidence probability is relatively big, the forecasting interval average bandwidth is much wider, the reason for which is that higher confidence level means higher cover probability. In order to make sure that the prediction results have higher completeness, average centering error should increase accordingly.

\section{Conclusion}

With the large-scale development of wind power, wind power prediction has been the key to solve the lots of problem existing in the wind power technology. In this paper, on the basis of traditional single point prediction, a wind power interval prediction model is set up considering multiple output characteristic of BP neural network. This model of interval prediction can avoid complicated nonlinear fitting and is easy to understand.

In this paper, in order to get better results in engineering application, a comprehensive optimization criterion containing PICP, PIANW, and PIACE is proposed, and improved PSO is adopted to train model parameters. Objective function takes interval band average bandwidth and average centering error into consideration. Improved
PSO-BPNN has an advantage in searching precision and is suitable to solve wind interval prediction.

The example shows that the proposed wind power interval prediction model has higher prediction reliability and practicability. It can describe the uncertainty of wind power more accurately. The predicted interval can meet the completeness requirements under specified confidence probability, which can provide power system dispatcher with decision basis.

\section{Acknowledgements}

The authors greatly acknowledge the support from National Natural Science Foundation of China (NSFC) (51477111) and National Key Research and Development Program of China (2016YFB0901104).

\section{References}

[1] B. Ernst, B. Oakleaf, M. L. Ahlstrom: "Predicting the wind," Power \& Energy Magazine. vol. 11, no. 2, pp. 79-89(2007).

[2] F. Scarlatache, G. Grigoras: "Influence of wind power plants on power systems operation," 2014 International Conference and Exposition on Electrical and Power Engineering. pp. 1010-1014(2014).

[3] A. Kusiak, H. Y. Zheng, Z. Song: "Short-Term prediction of wind farm power: a data mining approach," Energy Conversion. vol. 24, no. 1, pp. 125-136 (2009).

[4] J. W. Taylor, P. E. McSharry: "Wind power density forecasting using ensemble predictions and time series models," Energy Conversion. vol. 24, no. 3, pp. 775782(2009).

[5] C. Skittides, W. G. Fruh: "Wind forecasting using principal component analysis," Renewable Energy. vol. 69, pp. 365-374(2014).

[6] E. E. Elattar: "Prediction of wind power based on evolutionary optimized local general regression neural network," IET Generation, Transmission \& Distribution. vol.8, pp. 916-923(2014).

[7] A. Togelou, G. Sideratos N. D. Hatziargyriou: "Wind power forecasting in the absence of historical data," Sustainable Energy. vol. 3, no. 3, pp. 416-421(2012).

[8] Ramesh. Babu N, Arulmozhivarman. P. "Improving forecast accuracy of wind speed using wavelet transform and neural networks," Journal of Electrical Engineering \& Technology. vol. 8, no. 3, pp. 559564(2013)

[9] D. R. Chandra, M. S. Kumari, M. Sydulu, F. Grimaccia, M. Mussetta. "Adaptive wavelet neural network based wind speed forecasting studies," Journal of Electrical Engineering \& Technology. vol. 9, no. 6, pp. 1812-1821(2014) 
[10] J.W. Zeng, W. Qiao: "Support vector machine based short-term wind power forecasting," Power Systems Conference and Exposition (PSCE). pp. 1-8(2011).

[11] J. P. S. Catalao, H. M. I. Pousinho, V. M. F. Mendes: "Hybrid wavelet-PSO-ANFIS approach for shortterm wind power forecasting in Portugal," Sustainable Energy. vol. 2, no. 1, pp. 50-59(2010).

[12] P. Chen, H.Y. Chen, R Ye: "Chaotic wind speed series forecasting based on wavelet packet decomposition and support vector regression," International Power Electronics Conference (IPEC). pp. 256-261(2010).

[13] Y.Q. Liu., J. Shi, Y.P. Yang. "Short-term wind power prediction based on wavelet transform-support vector machine and statistic characteristics analysis," Industry Application. vol. 48, no. 4, pp. 1136-1141 (2012).

[14] A. U. Haque, P. A. Mandal: "Novel hybrid approach based on wavelet transform and fuzzy ARTMAP networks for predicting wind farm power production," Industry Application. vol. 49, no. 5, pp. 22532261 (2013).

[15] P. Pinson, G. Kariniotakis. "On-line assessment of prediction risk for wind power production forecasts," Proceeding of the European Wind Energy Conference and Exhibition. pp. 16-19(2003).

[16] H. Bludszuweit, N. Dominguez, A. Jose: "Statistical analysis of wind power forecast error," Power Systems. vol. 23, no. 3, pp. 983-991(2008).

[17] C. X. Wang, Z. X. Lu, Y. Qiao: "Short-term wind power prediction based on nonparametric regression model," Automation of Electric Power Systems. vol. 34, no. 16, pp. 78-82(2010).

[18] J. B. Sun, X. S. Wu, B. H. Zhang. "Wind power interval prediction based on nonparametric kernel density estimation," Water Resources and Power. vol. 31, no. 9, pp. 233-235(2013).

[19] A. Khosravi, S. Nahavandi, D. Creighton, A. Atiya. "Lower upper bound estimation method for construction of neural network-based prediction intervals," Neural Networks, vol. 22, no. 3, pp. 337-346(2011).

[20] D. Z. Huang, R. X. Gong, S. Gong. "Prediction of wind power by chaos and BP artificial neural networks approach based on Genetic Algorithm," Journal of Electrical Engineering \& Technology. vol. 10, no. 1, pp. 41-46(2015)

[21] Y. Song, Z.Q. Chen, Z.Z. Yuan: "New chaotic PSOBased neural network predictive control for nonlinear process," Neural Network. vol. 18, no. 2, pp. 595601(2007).

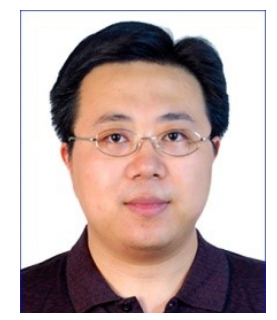

Jidong Wang He received his B.S. and M.S. degrees from Shandong University of Technology and Shandong University in 1999 and 2002 respectively. He received his Ph.D. degree from Tianjin University in 2005 . He worked as a post-doctoral from 2005 to 2007 in Tianjin University. $\mathrm{He}$ is now an Associate Professor of School of Electrical and Information Engineering in Tianjin University since 2007. His research interests include power quality, distributed generation system, microgrid and smart power consumption.

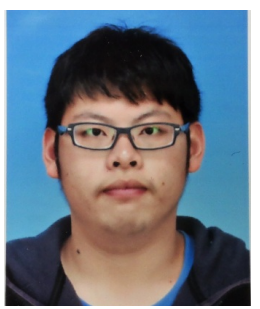

Kaijie Fang He received his B.S. degree from Tianjin University in 2015. $\mathrm{He}$ is now a postgraduate student in Tianjin University since 2015. His research interests include distributed generation and smart power consumption.

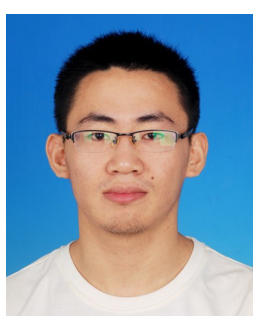

Wenjie Pang He received his B.S. degree and M.S. degree from Southeast University and Tianjin University in 2014 and 2017 rerspectively. His research interests include distributed generation and power quality.

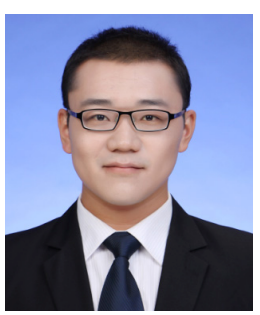

Jiawen Sun He received his B.S. and M.S. degree from Tianjin University in 2012 and 2015 respectively. His research interest is distributed generation forecast. 